\title{
Berufliche Gymnasien der sechsjährigen Aufbauform: Interessengeleitet durch die Sekundarstufe I?
}

\author{
Jochen Kramer $\mathbb{D} \cdot$ Katharina Hospach $•$ Ulrich Trautwein
}

Online publiziert: 31. Mai 2017

(C) Der/die Autor(en) 2017. Dieser Artikel ist eine Open-Access-Publikation.

Zusammenfassung In Baden-Württemberg gibt es einen deutschlandweit einzigartigen Weg zur allgemeinen Hochschulreife, der eine berufliche Spezialisierung ab der achten Klassenstufe beinhaltet. Dieser Weg wird von beruflichen Gymnasien der sechsjährigen Aufbauform (6BG) für leistungsstarke Schülerinnen und Schülern (SuS) von Real-, Haupt- und Werkrealschulen angeboten. Das 6BG kann aber auch von $\mathrm{SuS}$ besucht werden, die bis zur siebten Klassenstufe ein allgemeinbildendes Gymnasium besuchten und einen stärker ihren eigenen Interessen entsprechenden Weg zum Abitur bevorzugen. In der vorliegenden Studie wurde untersucht, inwiefern technische Gymnasien und Wirtschaftsgymnasien der sechsjährigen Aufbauform interessegeleitet gewählt werden (berufliche Interessen nach Holland 1997) und inwiefern sich fachspezifische Wertüberzeugungen in Mathematik, Physik, Deutsch und Englisch zwischen SuS an 6BG von SuS an allgemeinbildenden Gymnasien unterscheiden (Selektionseffekte). In einem zweiten Schritt wurde untersucht, ob sich bis zum Ende der achten Klassenstufe Interessen und Wertüberzeugungen unterschiedlich entwickeln, je nachdem welcher Gymnasialtyp gewählt wurde (Sozialisationseffekte). Befragt wurden SuS an je drei allgemeinbildenden Gymnasien, technischen Gymnasien und Wirtschaftsgymnasien $(N=443)$ im Schuljahr 2013/14. Die Ergebnisse sprechen dafür, dass technische Gymnasien interessegeleitet gewählt werden

\footnotetext{
Dr. J. Kramer $(\bowtie)$

Türkische Gemeinde in Baden-Württemberg, Reinsburgstraße 82, 70178 Stuttgart, Deutschland E-Mail: jochen.kramer@tgbw.de

K. Hospach

MTO Psychologische Forschung und Beratung GmbH, Schweickhardtstraße 5, 72072 Tübingen, Deutschland

E-Mail: k.hospach@mto.de
}

Prof. Dr. U. Trautwein

Hector-Institut für Empirische Bildungsforschung, Universität Tübingen,

Europastraße 6, 72072 Tübingen, Deutschland

E-Mail: ulrich.trautwein@uni-tuebingen.de 
und es ihnen gelingt, technisches Interesse während der achten Klassenstufe zu fördern. Bei Wirtschaftsgymnasien konnten dagegen für die berücksichtigten Variablen keine prononcierten Selektions- oder Sozialisationseffekte gefunden werden.

Schlüsselwörter Berufliche Gymnasien der sechsjährigen Aufbauform · Technische Gymnasien · Wirtschaftsgymnasien · Berufliche Interessen · Fachbezogene Motivation

\title{
Lower secondary-school and the vocational Gymnasium in 6 year form: The role of vocational interests
}

\begin{abstract}
In Baden-Wuerttemberg, there is a pathway to qualification for university entrance (Abitur) that is characterized by an vocational specialization from grade 8 to grade 13. It is the only pathway of this kind in Germany. That pathway is offered by vocational 6 year secondary schools (Berufliche Gymnasien der sechsjährigen Aufbauform, 6BG). 6BG can be attended by high-performing students from intermediate and general secondary school (Real-, Haupt-, Werkrealschulen) as well as students from general secondary schools (allgemeinbildenden Gymnasien) who prefer a pathway to Abitur that demonstrates a better fit with their particular predispositions and interests. In the present study, we focus the following questions: First, to what extent do students choose technical or commercial profiles of 6BG based on their vocational interests (Holland 1997) and to what extent do the beliefs regarding the subjects Mathematics, Physics, German and English differ between students of 6BG and students of general secondary schools (selection effects)? Second, do interests and beliefs develop differentially depending on the chosen type of secondary school (socialization effects)? In the study, grade 8 students $(N=443)$ from three schools per type (technical, commercial, and general secondary school) in the 2013/14 school year participated. Results show that students chose the technical 6BG based on interests and that these schools supports the promotion of technical interests during grade eight. Pronounced selection or socialization effects for the commercial 6BG could not be shown.
\end{abstract}

Keywords 6 year vocational secondary school - Commercial secondary school . Technical secondary school · Vocational interests $\cdot$ Vocational motivation

\section{Einleitung}

Baden-Württemberg kann auf eine lange Tradition beruflicher Gymnasien (BG) zurückblicken, auf denen die allgemeine Hochschulreife auf beruflich spezialisiertem Weg erworben werden kann. Dieser Weg ist sehr beliebt: Seit Jahrzehnten erwirbt rund ein Drittel der Schülerinnen und Schüler (SuS) das Abitur in Baden-Württemberg an einem BG (Kramer 2015). BG gelten deshalb als ein Musterbeispiel dafür, wie die traditionelle Trennung von ,allgemeiner“ und „beruflicher“ Bildung überwunden und das Bildungssystem durchlässiger gemacht werden kann (vgl. Watermann und Maaz 2006). Bis auf wenige Ausnahmen war dieser berufliche Weg zur 
allgemeinen Hochschulreife aber auf die letzten drei Jahre bis zum Abitur (Klassenstufen 11-13) begrenzt (BG der dreijährigen Aufbauform; 3BG). Seit dem Schuljahr 2012/2013 stehen die BGn schon ab der achten Klasse offen (BG der sechsjährigen Aufbauform; 6BG). Anlass für diesen Ausbau waren die von Schulen, Schulträgern und der Wirtschaft vorgebrachten Wünsche, durch frühzeitige fachspezifische Einblicke Fachkräfte von morgen besser zu fördern und den SuS damit schon in der Mittelstufe einen möglichst passgenauen und den eigenen Neigungen und Interessen entsprechenden Weg zur Hochschulreife zu ermöglichen (Kultusministeriums Baden-Württemberg 2011). 6BG sind damit ein Versuch, durch frühe fachliche Spezialisierung die Berufsorientierung der SuS zu fördern. Berufsorientierung ist ein Bildungsziel, dem in den letzten Jahren auch an allgemeinbildenden Schulen immer größere Aufmerksamkeit geschenkt wird (vgl. Dreer 2013).

Da Interessen für schulisches und außerschulisches Lernen eine zentrale Rolle spielen - sie sind maßgeblich für die Lernmotivation und damit die schulische und akademische Leistung (vgl. Krapp 1998) - werden Aufbau und Förderung stabiler Interessen als wichtiges Ziel schulischer Bildung angesehen. Dazu werden interessengeleitete Wahlmöglichkeiten angeboten, die unterschiedlich umfangreich ausgestaltet sind: An allgemeinbildenden Gymnasien erlauben die Wahl von Kernfächern oder Prüfungsthemen Wahlmöglichkeiten in einem gewissen Umfang. Die BG in Baden-Württemberg hingegen sind eigene Schulformen, die auf unterschiedliche fachliche Interessen zugeschnitten sind. Diese umfassende Möglichkeit der Profilbildung ist dazu geeignet, berufliche Interessen in der Sekundarstufe II zu fördern (Lüdtke und Trautwein 2004). Ob die Wahl eines BG bereits am Ende der siebten Klasse interessengeleitet erfolgt und ob sich die Wahl im nächsten Schuljahr förderlich auf die Interessenentwicklung und Lernmotivation auswirkt, ist dagegen bislang empirisch noch nicht untersucht worden.

Im vorliegenden Beitrag wird diesen Fragen nachgegangen, indem SuS miteinander verglichen werden, die ein 6BG der Fachrichtungen Technik (6TG) oder Wirtschaft $(6 \mathrm{WG})$ oder ein allgemeinbildendes Gymnasium (AG) besuchten. Betrachtet werden Interessenunterschiede und Unterschiede in fachbezogenen Wertüberzeugungen, die als wichtige Voraussetzung für Lernmotivation angesehen werden können. Es wird geprüft, ob sich die SuS im Hinblick darauf bereits zu Beginn der achten Klassenstufe unterscheiden und ob sich Interesse und Wertüberzeugungen bis zum Ende der achten Klassenstufe je nach Wahl des Gymnasialtyps unterschiedlich entwickeln.

\section{Berufliche Gymnasien der dreijährigen und sechsjährigen Aufbauform}

BG in Baden-Württemberg gibt es für die Fachrichtungen Wirtschaft, Technik, Biotechnologie, Ernährungs-, Agrar- sowie Sozial- und Gesundheitswissenschaft. Sie sind i. d. R. dreijährig, d. h. sie bestehen aus einer einjährigen Eingangsklasse (Klasse 11) und einer zweijährigen Kursstufe (Klassenstufen 1 und 2). Bereits seit den 1950er-Jahren gibt es aber auch schon ab Klasse 8 die Möglichkeit einen Weg zur Hochschulreife mit Spezialisierung in Wirtschaft zu wählen. Dazu standen zu- 
nächst fünf Wirtschaftsoberschulen zur Verfügung, die ab 1967 als WG weitergeführt wurden. Zum Schuljahr 2012/2013 wurde das Angebot an 6BG-Schulen mehr als verdoppelt und inhaltlich verbreitert, indem ein weiteres 6WG, vier 6TG und drei Gymnasien mit Schwerpunkt Ernährung, Soziales und Gesundheit (6ESG) hinzukamen.

An BG ist die Belegung eines Profilfaches vorgeschrieben, das der fachlichen Ausrichtung der Schule entspricht. Dabei wird ein starker Schwerpunkt auf Handlungswissen gelegt: in Technik beispielsweise auf das Erlernen und Erarbeiten fachrelevanter handwerklicher Grundfertigkeiten und die Durchführung von Laborversuchen; in Volks- und Betriebswirtschaftslehre sind Stunden für Projekte, Fallstudien, Plan- und Rollenspiele (sog. ,,handlungsorientierte Themenbearbeitung“, HOT) eingeplant, mit der methodische, personale und soziale Kompetenzen gefördert werden sollen (Kultusministerium Baden-Württemberg 2012a, 2012b). Das Profilfach ergänzt Deutsch, Mathematik und eine Fremdsprache, die - wie an AG - ebenfalls mit erhöhtem Anforderungsniveau belegt werden. Es wird zunächst vierstündig, in den Klassenstufen 12 und 13 sechsstündig unterrichtet. An BG unterrichten u.a. Lehrkräfte mit außerschulischer berufspraktischer Erfahrung. Auch die Bildungsstandards der anderen Fächer sind an BG an die jeweilige berufsfachliche Ausrichtung angepasst.

BG setzen damit in großem Umfang Forderungen um, die zur Förderung der Berufsorientierung vorgeschlagen werden: beispielsweise die altersgemäße, fächerübergreifende und im Rahmen des normalen Lehrstoffs verankerte Integration beruflicher Themen, ein höherer Lebensweltbezug und eine stärkere Kooperation mit schulexternen Fachkräften (Dreer 2013).

Ein weiterer wesentlicher Unterschied zwischen AG und BG ist die Regelschulzeit bis zum Abitur: Es wird an BG nach 13 Schuljahren (G9) erworben, während es an AG in Baden-Württemberg regelhaft nach 12 Schuljahren (G8) abgelegt wird. BG können von SuS besucht werden, die zuvor ein AG, eine Realschule oder eine Haupt- bzw. Werkrealschule besucht haben und bestimmte Zugangsvoraussetzungen erfüllen (vgl. Kultusministerium Baden-Württemberg 2015).

\section{Interesse und Motivation}

In der Interessen- und Motivationsforschung wurden theoretische Modelle entwickelt und empirisch überprüft, die es plausibel erscheinen lassen, dass eine frühe fachliche Spezialisierung, wie sie mit 6BG umgesetzt wird, zur Anregung und Strukturierung der beruflichen Identitätsbildung beitragen kann. Die Interessenforschung hat Versuche unternommen, die Gegenstände, auf die sich Interessen richten, zu systematisieren. Holland (1997) entwickelte dazu das RIASEC-Modell, das in der Berufs- und Persönlichkeitspsychologie große Verbreitung erfuhr. Das RIASEC-Modell unterscheidet sechs Personen- und Umwelttypen: solche, die technischhandwerkliche ( $R$ realistic), untersuchend-forschende (I investigativ), künstlerischsprachliche (A artistic), soziale ( $S$ social), führend-verkaufende (E enterprising) oder ordnend-verwaltende ( $C$ conventional) Tätigkeiten interessant finden bzw. erfordern. Die Profilfächer und die beruflichen Tätigkeiten, auf die 6BG vorbereiten, lassen 
sich diesen unterschiedlichen Interessensgegenständen zuordnen: Im 6TG werden stark handwerklich-technische (R) und untersuchend-forschende (d. h. naturwissenschaftliche; I) Inhalte thematisiert, im 6WG stark führend-verkaufende (E) und ordnend-verwaltende (in Form von Buchhaltung; C) Inhalte. Gemäß der KongruenzThese von Holland (1997) sollte eine passende, d. h. interessengeleitete Wahl eines 6BG schulische Zufriedenheit und Leistungen fördern, auch weil die Lernmotivation steigt.

In der Forschung zur Lernmotivation werden nach dem Grad ihrer Selbstbestimmung intrinsische Motivation (hohe Selbstbestimmung; eine Handlung wird um ihrer selbst Willen ausgeführt) und extrinsische Motivation (geringere Selbstbestimmung) unterschieden. Extrinsische Motivation ist dadurch gekennzeichnet, dass Ziele erreicht werden sollen, die außerhalb der eigentlichen Handlung liegen, beispielsweise eine gute Schulnote (Daniels 2008). Lernmotivation wird auch als das Produkt aus Erwartungs- und Wertüberzeugungen angesehen (vgl. Rheinberg 1981). Nach Eccles (2005) werden bei der subjektiven Einschätzung des Anreizwertes einer erfolgreichen Aufgabenbewältigung der intrinsische Wert (intrinsic value), die Wichtigkeit (attainment value), die Nützlichkeit (utility value) und die Kosten (cost) berücksichtigt. Unterricht kann sowohl die extrinsischen als auch intrinsischen Komponenten von Lernmotivation fördern.

Das 6BG stellt eine Lernumgebung dar, die potenziell die Lernmotivation über eine hohe Interessenpassung in besonderem Maße fördern kann. Damit diese förderlichen Wirkungen schon in Klasse 8 an 6BG einsetzen können, ist eine interessengeleitete Wahl des 6BG Voraussetzung, die nur bei weit fortgeschrittener Interessenentwicklung bis zum Ende der siebten Klasse erfolgen kann. Todt und Schreiber (1998) entwickelten ein Stufenmodell zur Beschreibung der Interessenentwicklung, in dem sie Theorien verschiedener Autorinnen und Autoren (Kohlberg 1967; Barnett 1975; Travers 1978; Gottfredson 1981) integrierten. Das Modell geht davon aus, dass zunächst eine universelle Interessiertheit an der materiellen und sozialen Umwelt besteht, die sich durch nach und nach entwickelnde Geschlechtsrollenvorstellungen ( $a b$ einem Alter von etwa drei Jahren), Fähigkeitsselbstkonzepte (ca. ab sechs), Vorstellungen von der eigenen Rolle, die man später in der Gesellschaft einnehmen möchte (ca. ab neun), und der persönlichen Zukunft (ab 15) zunehmend ausdifferenziert.

Die Ausdifferenzierung fachlicher Interessen in der Jugend bedeutet, dass die SuS ihr Interesse zunehmend auf weniger Fächer richten. In diesen Fächern bleibt das Interesse stabil oder steigt sogar an, während es in anderen Fächern abnimmt. Gottfried et al. (2001) beobachteten einen besonders starken Rückgang der mittelhohen Fachinteressen zwischen der 5. und 10. Klassenstufe in Mathematik sowie einen etwas geringeren in Naturwissenschaften und Englisch. In gesellschaftswissenschaftlichen Fächern und Geschichte stellten sie keine Abnahme der Interessen fest.

Häußler und Hoffmann (1995) beobachteten eine Abnahme des Interesses an physikalischen Themen und am Fach Physik von der 5. bis zur 10. Klassenstufe, die bei Mädchen deutlich stärker ausfiel als bei Jungen, obwohl Mädchen in der 5. Klassenstufe schon geringer an Physik interessiert waren. Die Interessenabnahme bei Mädchen konnte aber aufgehalten werden, wenn physikalische Themen mit 
Bezug zur Biologie behandelt wurden. Häußler und Hoffmann empfahlen daher eine Einbettung der Lerninhalte in für die SuS interessante alltags- und anwendungsnahe Kontexte. Köller et al. (2006) zeigten außerdem, dass auch die Leistungskurswahl interessefördernde Effekte haben kann.

Entsprechend kann davon ausgegangen werden, dass sich die fachlichen Interessen bis zum Ende der 7. Klasse schon so weit ausdifferenziert haben, dass eine interessengeleitete Schulwahl erfolgen kann. Es kann auch davon ausgegangen werden, dass sich diese förderlich auf die Entwicklung des Interesses an den Schwerpunktfächern der 6BG auswirkt. Das muss aber nicht bedeuten, dass die entsprechenden fachbezogenen Interessen zunehmen. Möglicherweise ist ein positiver Effekt schon darin zu sehen, dass eine Abnahme fachspezifischer Interessen verhindert oder zumindest vermindert werden kann.

\section{Schulwahlverhalten}

Bei den bisherigen Ausführungen wurde unterstellt, dass die Wahl eines 6BG gemäß dem Interesse der Schülerin oder des Schülers erfolgt. Die Forschung zum Schulwahlverhalten zeigt jedoch, dass die Wahl einer Schule von unterschiedlichen Determinanten abhängt. Sie wird auch nicht (allein) von den SuS selbst bestimmt, sondern maßgeblich von deren Eltern beeinflusst. Als zentrale Einflussfaktoren auf die Schulwahlentscheidung werden das Schulprofil - zu dem die fachliche Ausrichtung zählt -, die Qualität der Schule, die soziodemographische Zusammensetzung der Schule und die Schulatmosphäre beschrieben. Zusätzlich zu diesen Determinanten, die als Präferenzen verstanden werden können, sind der sozioökonomische Status der Eltern und die Lage der Schule wesentliche Restriktionen, die bei der Schulwahl berücksichtigt werden (vgl. Suter 2013).

Der Rational Choice Ansatz geht davon aus, dass sich die Familie aktiv mit der Schulwahl auseinandersetzt, sich informiert (z. B. über das Angebot der 6BG und die Interessen der Schülerin oder des Schülers) und darauf basierend eine rationale Entscheidung trifft. Dass diese Annahmen häufig zu kurz greifen, ist offensichtlich: Familien folgen nicht stringent einer Rationalität, sondern berücksichtigen auch persönliche Wertvorstellungen, Vorlieben und Wünsche sowie Meinungen und Informationen aus ihrem sozialen Umfeld; sie treffen Entscheidungen, die auch auf Willkür basieren, oder Nicht-Entscheidungen, ziehen also beispielsweise die Wahl eines 6BG bewusst oder unbewusst gar nicht in Betracht. Die Kulturkapitaltheorie, die auf Bourdieu (1970) zurück geht, berücksichtigt diese nicht rationalen Wahlaspekte. Sie zeigt auf, dass die Schulwahl ein sozialer Prozess ist, der auch beeinflusst wird vom gesellschaftlichen Milieu und dem sozialen Umfeld der Familien. Dieser Aspekt ist für die 6BG-Wahl aus zwei Gründen relevant. Zum einen unterscheiden sich die Schulformen in der Zusammensetzung ihrer Schülerschaft im Hinblick auf den sozioökonomischen Status, das kulturelle Kapital bzw. die gesellschaftlichen Milieus (vgl. Maaz et al. 2011). Zum anderen ist es nicht zwingend, sich mit der Wahl eines 6BG zu beschäftigen, da ein Schulwechsel nach der siebten Klassenstufe nicht erforderlich ist. 
Gemäß dem Rational Choice Ansatz werden Schulwahlentscheidungen basierend auf Kosten-Nutzenüberlegungen und Einschätzungen der Erfolgswahrscheinlichkeit so getroffen, dass ein maximaler Nutzen für die Familien erzielt wird. Dabei können auch der situative Kontext und strukturelle Zweckmäßigkeiten und Restriktionen berücksichtigt werden (vgl. Becker 1982). Demgemäß sollten die Interessen der SuS bei der Schulwahl berücksichtigt werden, da sie die Erfolgswahrscheinlichkeit durch die Steigerung der fachspezifischen Bildungsmotivation und damit vermutlich der Leistungen beeinflussen.

\section{Empirische Befunde zu Interessenunterschieden zwischen Schülerinnen und Schülern fachlich unterschiedlich spezialisierter Schultypen}

Inwiefern sich SuS, die fachlich unterschiedlich spezialisierte Schultypen auf dem Weg zur Hochschulreife besuchen, in ihren fachspezifischen Interessen unterscheiden, wurde bereits in Österreich (Eder 1988; Bergmann und Eder 1992; Eder und Reiter 2002) und in Baden-Württemberg (Lüdtke und Trautwein 2004) untersucht. Als Rahmenmodell der Interessenorientierungen wurde in beiden Fällen das RIASEC-Modell verwendet.

In Österreich gibt es wie in Baden-Württemberg die Möglichkeit, die gymnasiale Oberstufe auf einem fachlich spezialisierten Weg zu begehen. Eder (1988) zeigte, dass SuS an musisch ausgerichteten Schulen stark an künstlerisch-sprachlichen und sozialen Tätigkeiten interessiert waren, wohingegen bei SuS an naturwissenschaftlich ausgerichteten Schulen das Interesse an intellektuell-forschenden und unternehmerischen Tätigkeiten besonders ausgeprägt war. In einer weiteren Stichprobe fanden Bergmann und Eder (1992) dazu passende Unterschiede zwischen SuS an naturwissenschaftlichen Schulen (starke praktisch-technische Orientierung) und SuS an musischen Schulen (starke soziale Orientierung). Eine hohe Person-Umwelt-Passung (Kongruenz) ging mit besseren Noten, geringerer Devianz und weniger starkem Belastungserleben einher (Eder 1988). Entsprechende Effekte konnten auch im Rahmen einer österreichischen Ergänzung zur PISA-2000-Studie nachgewiesen werden: Hier zeigten sich positive Zusammenhänge von der Interessenkongruenz mit Noten, Leistungen in standardisierten Leistungstests und Wohlbefinden (Eder und Reiter 2002).

$\mathrm{Ob}$ sich baden-württembergische Abiturientinnen und Abiturienten an BG in ihren Interessen von denjenigen an AG unterscheiden, haben Lüdtke und Trautwein (2004) im Rahmen der TOSCA-Studie (Köller et al. 2004) untersucht. Die Ergebnisse fielen erwartungskonform aus: $\mathrm{SuS}$ an TG waren stark praktisch-technisch und untersuchend-forschend interessiert, SuS an WG waren stark führend-verkaufend und ordnend-verwaltend interessiert. Auch die Interessenausprägungen an den anderen BG-Formen entsprachen weitgehend den Erwartungen. 


\section{Fragestellungen}

Die empirischen Befunde sprechen dafür, dass BG in der Sekundarstufe II zu einer hohen Interessenkongruenz beitragen können. Offene Fragen sind hingegen, inwieweit diese Interessenkongruenz dadurch zustande kommt, dass die SuS bereits das passende BG gewählt haben (Selektionseffekt) oder inwieweit erst der Besuch des BG ihr Interesse geprägt hat (Sozialisationseffekt). Mit der Untersuchung dieser Fragen lässt sich auch klären, ob die Befunde zu BG aus der Sekundarstufe II auf jüngere $\mathrm{SuS}$ in der achten Klasse generalisierbar sind. Möglicherweise können oder wollen jüngere SuS ihre Interessen bei der Wahlentscheidung noch nicht so geltend machen, wie es ältere SuS tun. Auch ist während der Sekundarstufe I i.d. R. kein Übergang in eine andere Schulform vorgesehen. Deshalb stellen sich viele SuS am Ende der siebten Klasse die Frage gar nicht, ob sie ein BG besuchen sollen oder nicht.

Antworten auf die eben aufgeworfenen Fragen können mit vorliegender Studie gegeben werden. Diese basiert auf einem Vergleich von SuS, die auf einem 6TG, einem 6WG oder einem AG unterrichtet wurden und zu Beginn und zum Ende der achten Klassenstufe zu ihren beruflichen Interessen und fachbezogenen Wertüberzeugungen befragt werden konnten. Die fachbezogenen Wertüberzeugungen umfassen Einschätzungen des intrinsischen Wertes eines Faches, seiner Wichtigkeit und Nützlichkeit sowie der mentalen Kosten, die durch die Beschäftigung mit ihm entstehen.

Dabei wurde davon ausgegangen, dass 6TG besonders attraktiv für SuS sind, die bereits über ausgeprägte handwerklich-technische und untersuchend-forschende Interessen und hohe Wertüberzeugungen im Hinblick auf die Fächer Mathematik und Physik verfügen. Ferner davon, dass 6WG besonders attraktiv sind für $\mathrm{SuS}$ mit ausgeprägten führend-verkaufenden und ordnend-verwaltenden Interessen und hohen Wertüberzeugungen im Hinblick auf das Fach Mathematik (Selektionseffekte). Durch den Besuch des 6BG bis zum Ende der achten Klasse sollten diese fachlichen Interessen und Wertüberzeugungen gefördert werden (Sozialisationseffekte). Außerdem werden künstlerisch-sprachliche und soziale Interessen und die Wertüberzeugungen im Hinblick auf die Fächer Deutsch und Englisch mitbetrachtet - aber ohne konkrete Annahmen damit zu verbinden.

Geprüft werden folgende Hypothesen zu Selektions- und Sozialisationseffekten:

1 Selektion technisches Interesse. SuS zu Beginn des 6TG (Anfang achte Klasse) finden handwerklich-technische und untersuchend-forschende berufliche Tätigkeiten interessanter als $\mathrm{SuS}$ in 6WG oder AG.

2 Selektion wirtschaftliches Interesse. SuS zu Beginn des 6WG (Anfang achte Klasse) finden führend-verkaufende und ordnend-verwaltende berufliche Tätigkeiten interessanter als $\mathrm{SuS}$ in 6TG oder AG.

3 Selektion Wertüberzeugungen Mathematik und Physik. SuS zu Beginn des 6TG (Anfang achte Klasse) haben höhere fachbezogene Wertüberzeugungen zu Mathematik als SuS in AG und höhere in Physik als SuS in 6WG und AG. 
4 Sozialisation technisches Interesse. Die handwerklich-technischen und untersuchend-forschenden Interessen der SuS an 6TG entwickeln sich bis zum Ende der achten Klasse positiv. D. h. sie steigen im Vergleich zu denjenigen der SuS an 6WG oder an AG an oder verringern sich weniger stark.

5 Sozialisation wirtschaftliches Interesse. Die führend-verkaufenden und ordnend-verwaltenden Interessen der SuS an 6WG entwickeln sich bis zum Ende der achten Klasse positiv. D. h. sie steigen im Vergleich zu denjenigen der SuS an 6TG oder AG an oder verringern sich weniger stark.

6 Sozialisation Wertüberzeugungen Mathematik und Physik. Die fachbezogenen Wertüberzeugungen zu Mathematik und Physik der SuS an 6TG entwickeln sich bis zum Ende der achten Klasse positiv. D. h. sie steigen im Vergleich zu denjenigen der SuS an 6WG (Physik) oder AG (Mathematik und Physik) an oder verringern sich weniger stark.

\section{Methode}

\subsection{Teilnehmerinnen und -teilnehmer}

Grundlage der vorliegenden Untersuchung ist die Studie „Entwicklung von Motivationsprofilen im Schulvergleich“ (6BG-Studie), welche im Schuljahr 2013/2014 vom Hector-Institut für Empirische Bildungsforschung der Universität Tübingen durchgeführt wurde. Die Rekrutierung der Stichprobe erfolgte in Kooperation mit dem Beruflichen Schulzentrum Bietigheim-Bissingen, das die Studie mitinitiierte, durch direkte Ansprache von 6TG und 6WG sowie AG im Norden Baden-Württembergs. Die Datenerhebungen fanden zu Beginn (T1: Oktober/November 2013) und am Ende (T2: Juni/Juli 2014) des Schuljahres 2013/14 statt. Sie wurden von geschulten Testleiterinnen und Testleitern im Klassenverband während der Schulzeit durchgeführt und nahmen jeweils eine Schulstunde in Anspruch. Über den Stichprobenumfang und die Teilnahmequoten insgesamt und getrennt nach Schultyp informiert Tab. 1.

Tab. 1 Stichprobenumfang und Teilnahmequote

\begin{tabular}{|c|c|c|c|c|c|c|c|c|c|c|c|c|}
\hline & \multicolumn{3}{|l|}{$\mathrm{AG}$} & \multicolumn{3}{|l|}{$6 \mathrm{WG}$} & \multicolumn{3}{|l|}{$6 \mathrm{TG}$} & \multicolumn{3}{|c|}{ Gesamt } \\
\hline & $\mathrm{T} 1$ & $\mathrm{~T} 2$ & $\begin{array}{l}\text { Beide } \\
\mathrm{T}\end{array}$ & $\mathrm{T} 1$ & $\mathrm{~T} 2$ & $\begin{array}{l}\text { Beide } \\
\mathrm{T}\end{array}$ & $\mathrm{T} 1$ & $\mathrm{~T} 2$ & $\begin{array}{l}\text { Beide } \\
\mathrm{T}\end{array}$ & $\mathrm{T} 1$ & $\mathrm{~T} 2$ & $\begin{array}{l}\text { Beide } \\
\mathrm{T}\end{array}$ \\
\hline Schulen & 3 & 3 & 3 & 3 & 3 & 3 & 3 & 3 & 3 & 9 & 9 & 9 \\
\hline Klassen & 10 & 10 & 10 & 8 & 8 & 8 & 6 & 6 & 6 & 24 & 24 & 24 \\
\hline $\begin{array}{l}\text { Eingeladene } \\
\text { SuS }\end{array}$ & 270 & 268 & 268 & 191 & 190 & 190 & 131 & 129 & 129 & 592 & 587 & 587 \\
\hline $\begin{array}{l}\text { Teilnehmende } \\
\text { SuS }\end{array}$ & 167 & 165 & 156 & 131 & 136 & 125 & 127 & 116 & 116 & 425 & 417 & 397 \\
\hline $\begin{array}{l}\text { Teilnahme- } \\
\text { quote }(\%)\end{array}$ & 61,9 & 61,6 & 58,2 & 68,6 & 71,6 & 65,8 & 96,9 & 89,9 & 89,9 & 71,8 & 71,0 & 67,6 \\
\hline
\end{tabular}


Tab. 2 Geschlecht, Bildungshintergrund der Eltern, Herkunftsschulart und kognitive Grundfähigkeiten der Teilnehmerinnen und Teilnehmer

\begin{tabular}{|c|c|c|c|c|}
\hline & $\mathrm{AG}$ & $6 \mathrm{WG}$ & $6 \mathrm{TG}$ & Inferenzstatistik \\
\hline \multicolumn{5}{|c|}{ Prozentanteile (Zellenprozent) } \\
\hline Anteil weiblich & $53,7 \%$ & $50,0 \%$ & $14,8 \%$ & $\begin{array}{l}\chi^{2}[2, n=435]=50,77 \\
\mathrm{CC}=0,34 * * *\end{array}$ \\
\hline Gymnasialanteil Eltern & $58,2 \%$ & $56,8 \%$ & $73,3 \%$ & $\begin{array}{l}\chi^{2}[2, n=435]=8,78 \\
\mathrm{CC}=0,14^{*}\end{array}$ \\
\hline Anteil Realschule in K7 & $0,0 \%$ & $42,8 \%$ & $52,5 \%$ & $\begin{array}{l}\chi^{2}[2, n=435]=118,43 \\
\mathrm{CC}=0,46^{* * *}\end{array}$ \\
\hline \multicolumn{5}{|c|}{ Kognitive Grundfähigkeiten M (SD) } \\
\hline Wortanalogien-Skala & $12,5(2,9)$ & $10,2(3,3)$ & $12,0(3,3)$ & $\mathrm{F}=20,77 * * *$ \\
\hline Figurenanalogien-Skala & $19,0(4,7)$ & $16,0(6,2)$ & $16,8(6,6)$ & $\mathrm{F}=11,14 * * *$ \\
\hline
\end{tabular}

$C C$ Kontingenzkoeffizient

$* p<0,05 ; * * * p<0,001$

Die höchste Beteiligungsquote $(95,8 \%$ ) wurde im Beruflichen Schulzentrum Bietigheim-Bissingen erreicht. Mit Sensitivitätsanalysen wurde geprüft, ob diese hohe Beteiligung einen verzerrenden Einfluss auf die Ergebnisse hatte. Dies war nicht der Fall. Lediglich acht SuS gaben an, in der 7. Klassenstufe eine Haupt- oder Werkrealschule besucht zu haben. Diese acht wurden von den weiteren Analysen ausgeschlossen, weshalb die Aussagekraft der Befunde auf SuS beschränkt ist, die in der 7. Klassenstufe eine Realschule oder ein Gymnasium besuchten.

Im Hinblick auf Geschlecht, Bildungshintergrund der Eltern, Herkunftsschularten und kognitive Grundfähigkeiten unterschieden sich die SuS an den drei Schultypen (vgl. Tab. 2): Während der Anteil von Schülerinnen an AG und 6WG ausgeglichen war, war er mit $14,8 \%$ an 6TG deutlich geringer. Etwas mehr als die Hälfte der SuS der AG und 6WG hatten ein Elternteil, das ein Gymnasium besuchte, an 6TG waren es mit ca. drei Viertel deutlich mehr. Nimmt man die Herkunftsschulart der $\mathrm{SuS}$ in den Blick, wechselten von den SuS am 6WG und 6TG ein großer Teil nach Klasse 7 von der Realschule auf die 6BG. Der Anteil der ehemaligen Real-SuS auf den AG lag bei 0,0\%. Erwartungsgemäß unterschieden sich die SuS in ihren kognitiven Grundfähigkeiten: $\mathrm{SuS}$ an $\mathrm{AG}$ erzielten etwas bessere Werte als $\mathrm{SuS}$ an 6BG (zur Operationalisierung der kognitiven Grundfähigkeiten: s. u. Instrumente).

\subsection{Instrumente}

Berufliches Interesse. Die beruflichen Interessen wurden anhand des RIASECModells von Holland (1997) operationalisiert. Die Erfassung erfolgte mit einer Kurzfassung des Allgemeinen Interessens Struktur-Tests (AIST-R; Bergmann 2005) mit insgesamt 33 Items (5-7 Items pro Subskala). Die Befragten gaben auf einer fünfstufigen Likert-Skala $(1=$ gar nicht bis $5=s e h r)$ an, in welchem Ausmaß sie verschiedene schulische und berufliche Tätigkeiten interessieren. Die Interessenskalen wiesen gute interne Konsistenzen auf (Cronbachs $\alpha>0,68$; vgl. Tab. 3).

Fachbezogene Wertzuschreibungen. Die fachbezogenen Wertzuschreibungen wurden in den Fächern Mathematik, Physik, Deutsch und Englisch erhoben. Für die 
Tab. 3 Messinstrumente

\begin{tabular}{|c|c|c|c|c|}
\hline \multirow[t]{2}{*}{ Skala } & \multirow[t]{2}{*}{ Itembeispiel } & \multirow{2}{*}{$\begin{array}{l}\text { Anzahl } \\
\text { Items }\end{array}$} & \multicolumn{2}{|c|}{ Cronbachs $\alpha$} \\
\hline & & & $\mathrm{T} 1$ & $\mathrm{~T} 2$ \\
\hline \multicolumn{5}{|l|}{ Berufliche Interessen } \\
\hline $\begin{array}{l}\mathrm{R} \text { handwerklich-tech- } \\
\text { nisch }\end{array}$ & $\begin{array}{l}\text { Mit Maschinen oder technischen Geräten } \\
\text { arbeiten }\end{array}$ & 6 & 0,82 & 0,82 \\
\hline $\begin{array}{l}\text { I untersuchend-for- } \\
\text { schend }\end{array}$ & Etwas genau beobachten und analysieren & 7 & 0,78 & 0,81 \\
\hline $\begin{array}{l}\text { A künstlerisch-sprach- } \\
\text { lich }\end{array}$ & $\begin{array}{l}\text { Etwas nach künstlerischen Gesichtspunkten } \\
\text { gestalten }\end{array}$ & 5 & 0,73 & 0,79 \\
\hline S sozial & Jemanden unterrichten oder erziehen & 5 & 0,80 & 0,82 \\
\hline E führend-verkaufend & $\begin{array}{l}\text { Andere von etwas überzeugen oder etwas } \\
\text { veranlassen }\end{array}$ & 5 & 0,72 & 0,76 \\
\hline $\mathrm{C}$ ordnend-verwaltend & Dinge sammeln, ordnen oder verwalten & 5 & 0,69 & 0,77 \\
\hline \multicolumn{5}{|c|}{ Schulfachbezogene Wertüberzeugungen } \\
\hline Mathematik & & 47 & 0,91 & 0,91 \\
\hline Intrinsischer Wert & Mathematik macht mir Spaß & 4 & 0,95 & 0,94 \\
\hline Wichtigkeit & $\begin{array}{l}\text { Mir liegt viel daran, den Stoff in Mathematik } \\
\text { zu behalten }\end{array}$ & 10 & 0,92 & 0,93 \\
\hline Nützlichkeit & $\begin{array}{l}\text { Eine gute Note in Mathematik kann mir } \\
\text { später sehr nutzen }\end{array}$ & 22 & 0,94 & 0,94 \\
\hline $\operatorname{Kosten}(-)^{\mathrm{a}}$ & $\begin{array}{l}\text { Mich mit Mathematik zu beschäftigen ist } \\
\text { anstrengend }\end{array}$ & 11 & 0,91 & 0,94 \\
\hline Physik & Mir ist es wichtig, in Physik viel zu wissen & 5 & 0,89 & 0,88 \\
\hline Deutsch & Mir ist es wichtig, in Deutsch viel zu wissen & 5 & 0,84 & 0,86 \\
\hline Englisch & Mir ist es wichtig, in Englisch viel zu wissen & 5 & 0,84 & 0,85 \\
\hline
\end{tabular}

ahohe Kosteneinschätzung = geringe Wertüberzeugung

Wertzuschreibungen in Mathematik kam ein 47 Items umfassendes Instrument aus dem Projekt Motivationsförderung im Mathematikunterricht (MoMa; Gaspard et al. 2015) zum Einsatz, das es erlaubt, die vier Komponenten der Wertzuschreibung (intrinsischer Wert, Wichtigkeit, Nützlichkeit und Kosten) des Erwartungs-WertModells nach Eccles (2005) mit jeweils eigenen Skalen differenziert zu erfassen. Der intrinsische Wert des Faches wird im Instrument von Gaspard et al. mit vier Items operationalisiert. Die drei Komponenten Wichtigkeit, Nützlichkeit und Kosten werden mit mehr Items erfasst, da sie in mehrere Subfacetten unterteilt werden können: Die Wichtigkeit einer Aufgabe (z. B. einer mathematischen Aufgabe) kann auf die Bedeutung der Leistung (,Es ist mir wichtig, gut in Mathe zu sein“) oder auf die persönliche Bedeutung (,Mathe ist mir persönlich sehr wichtig“) bezogen sein. Die Skala zur persönlichen Wichtigkeit umfasst zehn Items. Die empfundene Nützlichkeit kann mit verschiedenen Lebensbereichen in Verbindung gebracht werden: mit der Schule, dem Alltag, dem sozialen Nutzen (z. B. bei peers), dem (späteren) Beruf oder dem generellen Nutzen für das weitere Leben. Die Skala zur Nützlichkeit umfasst 22 Items. Die Kosten einer Aufgabe können auf die Anstrengung an sich, die dabei erlebten Emotionen oder die Abwägung der Opportunitätskosten (,Ich muss viel aufgeben, um in Mathe gut zu sein") bezogen werden. Die Skala zu den Kosten umfasst elf Items. 
Die Wertzuschreibungen in den Fächern Deutsch, Englisch und Physik wurden anhand von ebenfalls im MoMa-Projekt eingesetzten fünf Items umfassenden Kurzskalen erhoben. Dabei werden die Komponenten des Erwartungs-Wert-Modells mit jeweils ein bis zwei Items abgebildet. Die Itemwortlaute sind für die Skalen zu den einzelnen Fächern bis auf die Fachbezeichnungen identisch. Die SuS wurden gebeten anzugeben, wie sehr sie den einzelnen Aussagen zu ihren Wertüberzeugungen zustimmen (vierstufig Likertskala von $1=$ stimmt gar nicht bis $4=$ stimmt genau).

Kognitive Grundfähigkeiten. Zur Messung der kognitiven Grundfähigkeiten wurden zwei Untertests aus dem Kognitiven Fähigkeitstest für 4.-12. Klassen, Revision (KFT 4-12 + R; Heller und Perleth 2000) eingesetzt: Wortanalogien und Figurenanalogien. Kognitive Grundfähigkeiten wurden nur zum ersten Messzeitpunkt erfasst.

\subsection{Statistische Analysen}

Zur Prüfung der Hypothesen wurden univariate Regressionsanalysen mit Mplus (Version 7; Muthén und Muthén 2012) berechnet. Für die Analysen der Selektionseffekte (Tab. 4) wurden die zu T1 gemessenen Interessen- und Motivationsvariablen zunächst als abhängig von den Kontrollvariablen (Geschlecht, Bildungshintergrund der Eltern und allgemeine kognitive Fähigkeiten, jeweils zentriert) betrachtet (Regressionsschritt 1). Im zweiten Regressionsschritt wurde zusätzlich die Zughörigkeit zum Schultyp als unabhängige Variable berücksichtigt (dummykodiert mit AG als Referenzkategorie). Berichtet werden die $b$-Koeffizienten der Schultyp-Dummyvariablen aus Schritt 2, die Auskunft darüber geben, um wie viele Punkte sich der Mittelwert der abhängigen Variable in der 6WG- bzw. 6TG-Gruppe von der AG-Gruppe bei mittlerer Ausprägung der Kontrollvariablen unterscheidet. Zusätzlich wurde $\Delta R^{2}$ berechnet, das den Anteil an der Varianz der abhängigen Variable angibt, der durch die zusätzliche Berücksichtigung des Schultyps in Schritt 2 aufgeklärt werden kann. Um auch den Unterschied zwischen den zwei beruflichen Gymnasialtypen einschätzen zu können, wurden die Regressionsanalysen mit 6TG als Referenzgruppe wiederholt.

Um die Frage zu beantworten, ob sich innerhalb der einzelnen Schultypen über das Schuljahr hinweg signifikante Änderungen in Interessen und Wertüberzeugungen zeigten, wurden Differenzwerte (T2 - T1) gebildet und auf Signifikanz geprüft. Um faire Vergleiche zu ermöglichen, die unbeeinflusst sind von unterschiedlichen Ausprägungen der Kontrollvariablen (Geschlecht, Bildungshintergrund der Eltern und allgemeine kognitive Fähigkeiten), wurden die Differenzwerte für die mittleren Ausprägungen dieser Variablen über alle Schultypen hinweg adjustiert ${ }^{1}$.

Für die Analysen der Sozialisationseffekte (Tab. 5) wurden die (nicht adjustierten) Differenzwerte auf dieselbe Weise regressionsanalytisch untersucht, die zur Analyse der Selektionseffekte genutzt wurde: Durchgeführt wurden wiederum zweischrittige Regressionsanalysen, mit Adjustierung der Kontrollvariablen in Schritt 1 und zusätz-

\footnotetext{
${ }^{1}$ Zur Berechnung der adjustierten Differenzwerte wurden die Ergebnisse der Regressionsanalysen zu Sozialisationseffekten genutzt: $D_{\text {adj., T2-T1 }}=$ Intercept $+b$-Koeffizient.
} 


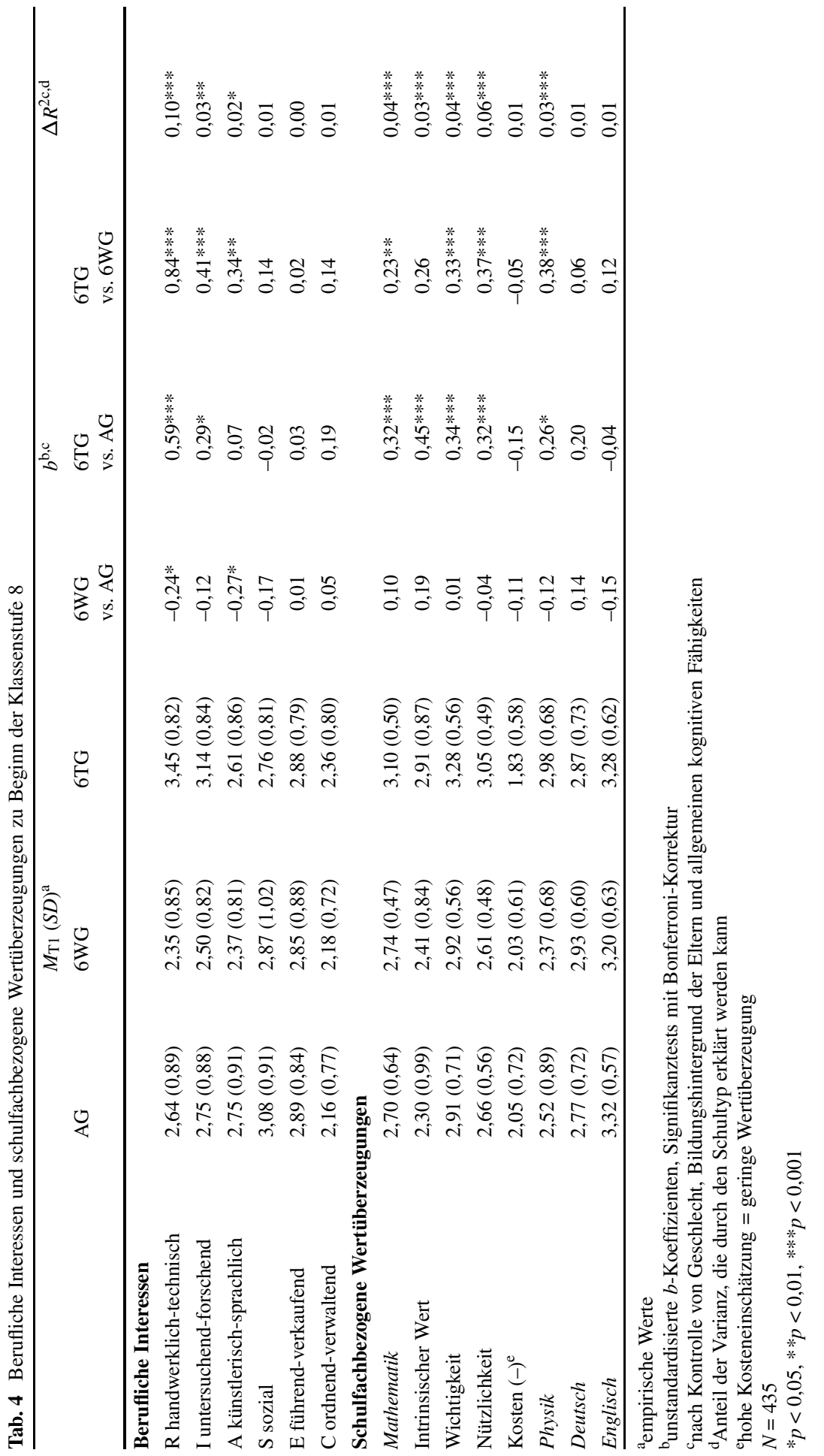




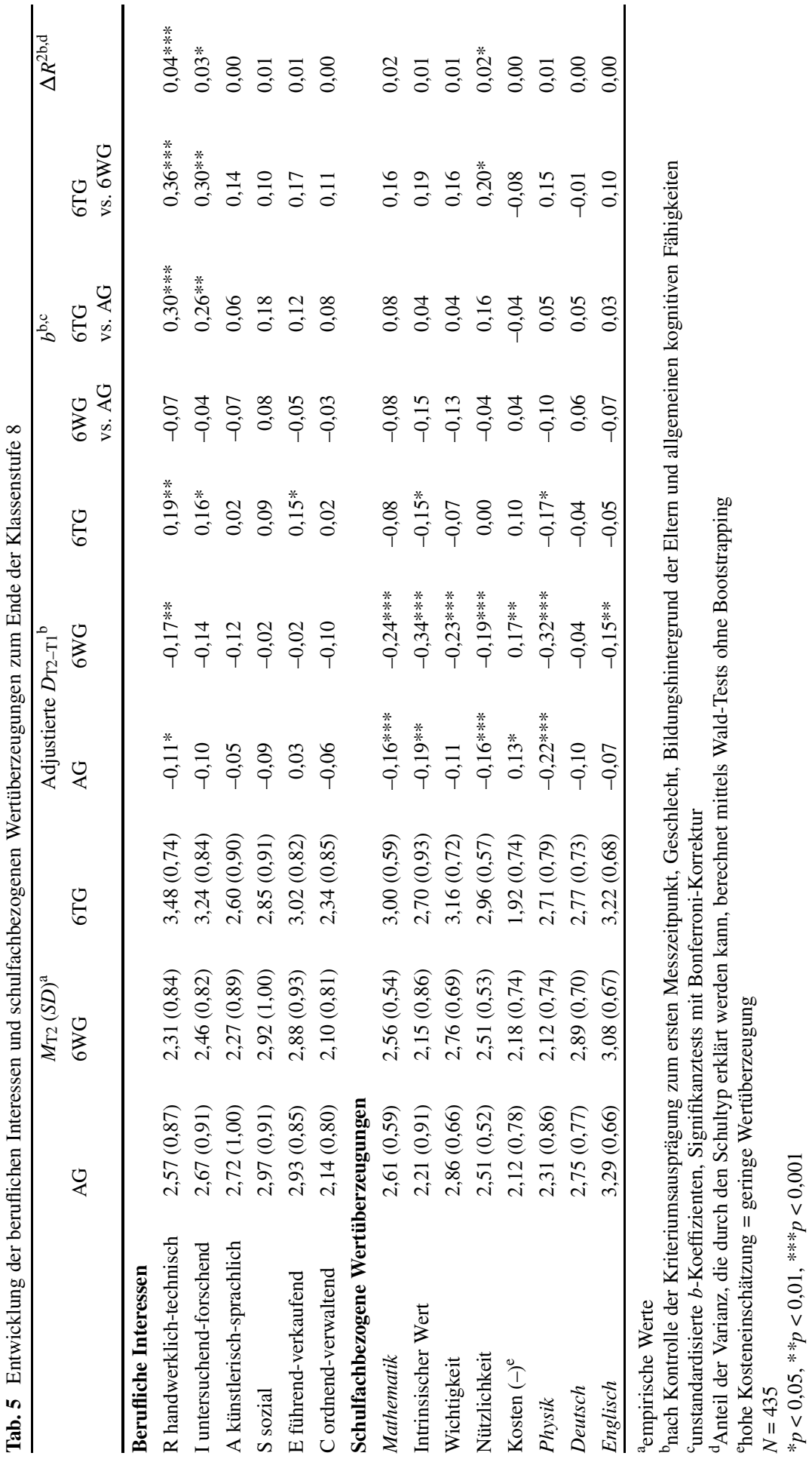


licher Berücksichtigung der dummy-kodierten Schultypen in Schritt 2. Auch diese Analysen wurden mit AG als Referenzkategorie durchgeführt und dann mit 6TG als Referenzkategorie wiederholt. Als Kontrollvariable wurde bei den Analysen zu den Sozialisationseffekten jedoch zusätzlich die Ausprägung der abhängigen Variable zum ersten Messzeitpunkt berücksichtigt. Die auf diese Art berechneten $b$-Koeffizienten sind damit auch fair im Hinblick auf unterschiedliche Ausgangsniveaus in den untersuchten Merkmalen zu Schuljahresbeginn. In anderen Worten: sie sind in Bezug auf die berücksichtigten Variablen unabhängig von den Selektionseffekten.

Um die Angaben aller Teilnehmerinnen und Teilnehmer auch bei fehlenden Werten auf einzelnen Variablen ${ }^{2}$ vollständig nutzen zu können, wurde bei allen Analysen das Full Information Maximum Likelihood-Verfahren (FIML; vgl. Lüdtke et al. 2007) eingesetzt. Zur Schätzung robuster Standardfehler wurde die Bootstrap-Methode mit 1000 Iterationen verwendet.

\section{Ergebnisse}

Die Ergebnisse der Regressionsanalysen zu den Selektionshypothesen sind in Tab. 4 zusammengestellt. Die Ergebnisse der Hypothesenprüfung veranschaulicht außerdem Abb. 1a.

Wie angenommen wiesen SuS an 6TG höhere handwerklich-technische und untersuchend-forschende Interessen auf als SuS an AG und 6WG (Hypothese 1). Erwartungskonträr zeichneten sich die $\mathrm{SuS}$ an $6 \mathrm{WG}$ jedoch nicht durch höhere führendverkaufende und ordnend-verwaltende Interessen aus als die SuS an AG oder an 6TG (Hypothese 2). Es wurden keine Hypothesen zu Selektionseffekten in Bezug auf künstlerisch-sprachliche und soziale Interessen formuliert. Es zeigte sich aber, dass die künstlerisch-sprachlichen Interessen der $\mathrm{SuS}$ an 6WG im Vergleich zu AG und 6TG geringer ausgeprägt waren. Die fachbezogenen Wertüberzeugungen in Mathematik und Physik unterschieden sich - wie vermutet wurde - deutlich zwischen SuS, die 6TG wählten, und den SuS an AG. Beide Fächer wurden von SuS an 6TG stärker subjektiv wertgeschätzt (Hypothese 3). Dies gilt auch im Vergleich von 6TG und 6WG. Die Wertüberzeugungen in Mathematik wurden mit einem differenzierteren Instrument erfasst, das auch Aussagen auf vier einzelnen Wertkomponenten zulässt. Die Komponenten Wichtigkeit und Nützlichkeit von Mathematik wurden von den SuS an 6TG höher eingeschätzt als an AG und 6WG. Außerdem war der intrinsische Wert an 6TG höher ausgeprägt als an AG. Keine Unterschiede zeigten sich in der Einschätzung der Kosten, die mit der Beschäftigung mit Mathematik verbunden sind. Ebenso fanden sich keine unterschiedlichen Wertzuschreibungen für die sprachlichen Fächer Deutsch und Englisch zwischen den drei Schultypen zum ersten Messzeitpunkt.

Vor der Prüfung der Sozialisationshypothesen wurde untersucht, ob sich während der achten Klassenstufe innerhalb der einzelnen Schultypen Veränderungen in den

\footnotetext{
2 Der Anteil fehlender Werte auf einzelnen Variablen liegt zwischen 10,6\% (handwerklich-technische, untersuchend-forschende, künstlerisch-sprachliche und soziale berufliche Interessen) und 31,7\% (mathematische Wertüberzeugungen insgesamt). Im Mittel fehlen 15,0\% der Werte pro Variable $(S D=7,0 \%)$.
} 


\section{a) Selektionseffekte}

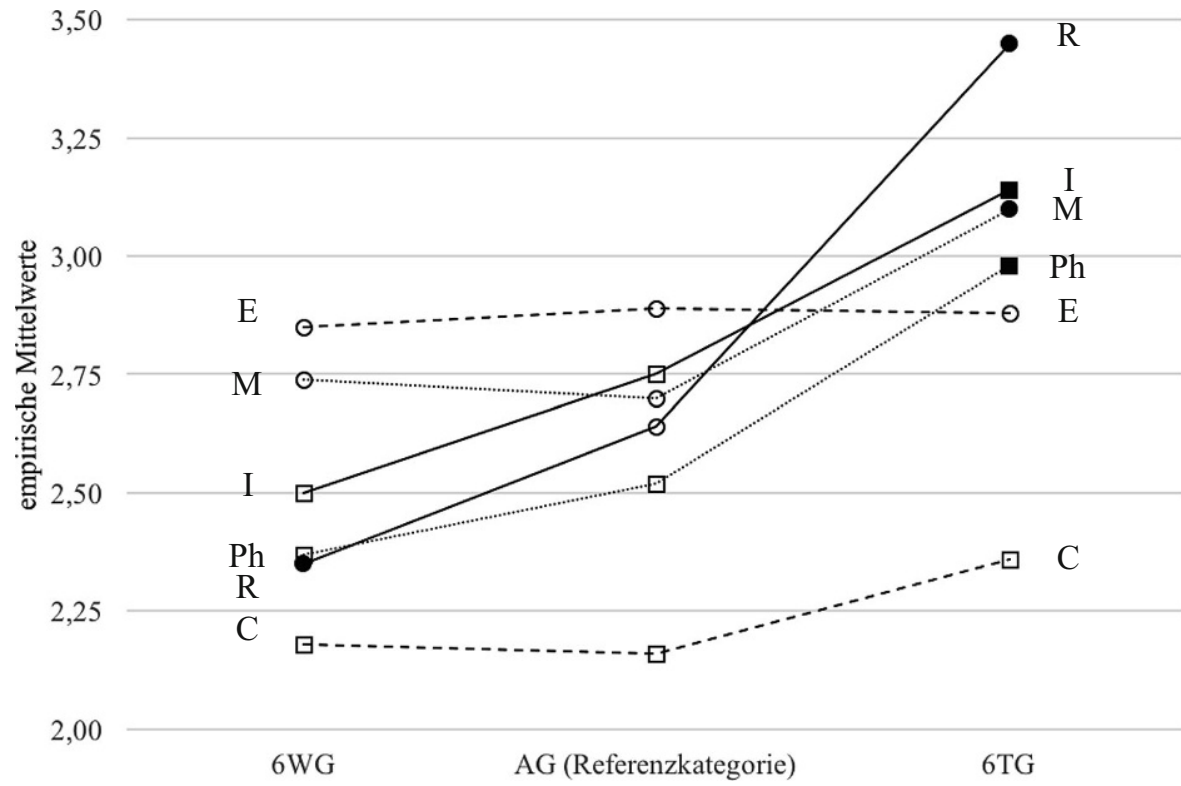

\section{b) Sozialisationseffekte}

0,3

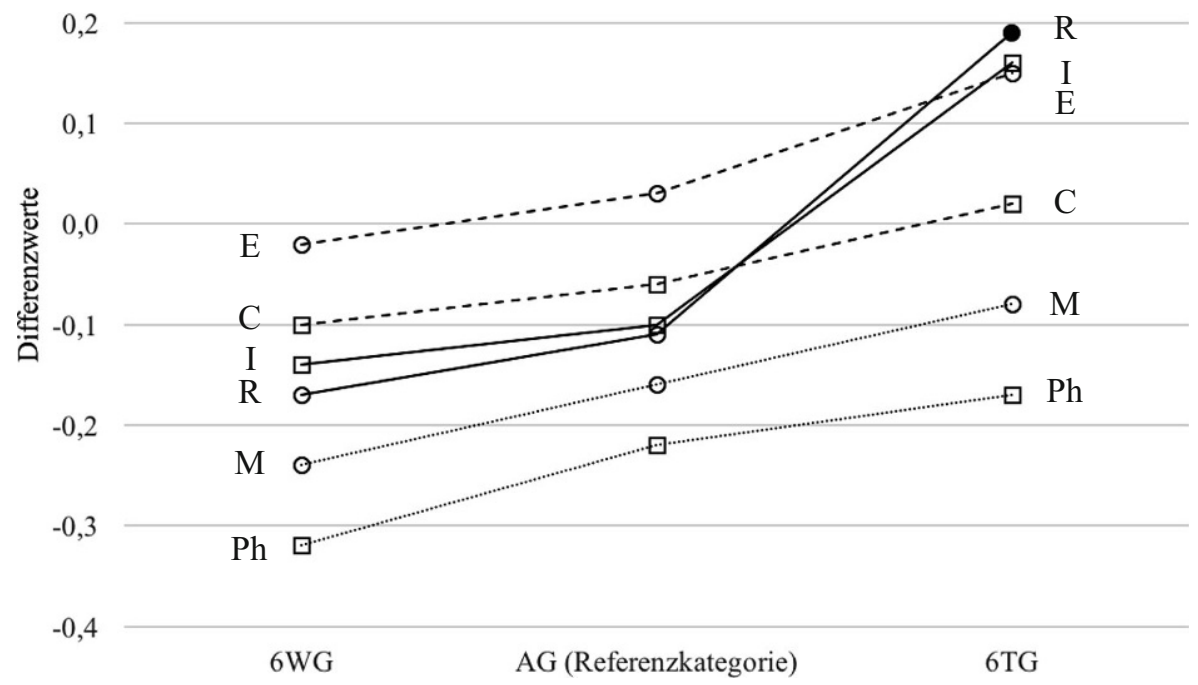

Abb. 1 Unterschiede zwischen den SuS der verschiedenen Schultypen ( $R$ handwerklich-technisches Interesse, $I$ untersuchend-forschendes Interesse, $E$ führend-verkaufendes Interesse, $C$ ordnend-verwaltendes Interesse, $M, P h$ Wertüberzeugungen in Mathematik und Physik; gefüllte Markierungen signifikante Unterschiede im Vergleich zur Referenzkategorie AG $(p<0,05))$ 
Interessenausprägungen und Wertüberzeugungen zeigten. Dafür wurden adjustierte Differenzwerte gebildet (vgl. Tab. 5 und Abb. 1b), die sich für die handwerklichtechnischen Interessen in allen Schultypen signifikant von Null unterschieden: Die handwerklich-technischen Interessen nahmen bei SuS an AG und $6 \mathrm{WG}$ ab, bei SuS an 6TG zu. Das untersuchend-forschende und führend-verkaufende Interesse nahm bei $\mathrm{SuS}$ an 6TG ebenfalls $\mathrm{zu}$, nicht aber bei $\mathrm{SuS}$ an AG und 6WG. Die anderen beruflichen Interessen änderten sich nicht bedeutsam während der achten Klassenstufe. Die schulfachbezogenen Wertüberzeugungen in Physik nahmen zum Schuljahresende in allen Schultypen ab. Für Mathematik war dies bei SuS an AG und 6WG ebenfalls der Fall. SuS an AG schätzten den intrinsischen Wert, die Nützlichkeit und die Kosten für die Beschäftigung mit Mathematik ungünstiger ein als zu Schuljahresbeginn, SuS an 6WG darüber hinaus auch die Wichtigkeit von Mathematik. Bei SuS an 6TG nahm der intrinsische Wert von Mathematik ab. Die Wertüberzeugungen für die sprachlichen Fächer änderten sich nicht bedeutsam, mit Ausnahme von Englisch an 6WG (abnehmende Werteinschätzung).

Regressionsanalytisch wurde geprüft, ob sich die Entwicklung der Interessenund Wertüberzeugungen zwischen den Schultypen differenziell unterschied (Sozialisationseffekte). Die Ergebnisse dieser Analysen enthalten die Spalten mit den $b$ - und $\Delta R^{2}$-Werten in Tab. 5. Erwartungskonform entwickelte sich das handwerklich-technische und untersuchend-forschende Interesse der SuS an 6TG positiv, d. h. es nahm im Vergleich zur AG- und 6WG-Gruppe zu (Hypothese 4). Keine differenzielle Entwicklung war für die führend-verkaufenden und ordnend-verwaltenden Interessen nachweisbar. Hypothese 5, in der eine positive Entwicklung für SuS an 6WG angenommen wurde, bewährte sich damit nicht. Die Zunahme im führend-verkaufenden Interesse an 6TG unterscheidet sich nicht bedeutsam von der Interessenentwicklung an AG und 6WG. Auch in den künstlerisch-sprachlichen und sozialen Interessen waren keine differenziellen Effekte auszumachen. Das gilt ebenso für die fachbezogenen Wertüberzeugen der vier untersuchten Fächer. Auch Hypothese 6, in der eine positive Entwicklung der Wertüberzeugungen für Mathematik und Physik an 6TG angenommen wurde, bewährte sich damit nicht. Bei einzelner Betrachtung der vier Komponenten mathematischer Wertüberzeugungen, fällt die Nützlichkeitseinschätzung von Mathematik auf: Sie entwickelte sich in der 6TG-Gruppe positiver (keine Abnahme) im Vergleich zur 6WG-Gruppe (Abnahme).

\section{Diskussion}

Ziel der Studie war es zu prüfen, ob berufliche Gymnasien, die bereits mit der achten Klassenstufe einsetzen, interessengeleitet gewählt werden und sich ihr Besuch während der achten Klassenstufe förderlich auf fachspezifische Interessen und Wertüberzeugungen auswirkt. Diese Fragestellung wurde mit Daten untersucht, die im Schuljahr 2013/14 an jeweils drei AG, TG und WG Baden-Württembergs erhoben wurden. Im Folgenden werden die Ergebnisse zusammengefasst, die Grenzen der Studie aufgezeigt und ein Fazit im Hinblick auf den Beitrag gezogen, den 6BG zur Berufsorientierung leisten. 


\subsection{Zentrale Befunde}

Die Befunde der vorliegenden Studie sprechen dafür, dass es 6TG gelingt, bereits für die achte Klassenstufe SuS zu gewinnen, die handwerklich-technisch und naturwissenschaftlich untersuchend-forschend stark interessiert sind und den Fächern Mathematik und Physik einen hohen Wert zuschreiben. Diese positiven Wertüberzeugungen können als eine wichtige Voraussetzung für die Lernmotivation in diesen Fächern angesehen werden. Obwohl die SuS in diesen Merkmalen zu Beginn des 6TG über höhere Werte verfügen als diejenigen an AG und 6WG, steigern sich handwerklich-technische und untersuchend-forschende Interessen der SuS an 6TG noch bis zum Ende der achten Klassenstufe. Dieser Zugewinn an technischem Interesse ging nicht zu Lasten anderer beruflicher Interessen.

Im Gegensatz dazu konnte für SuS zu Beginn des 6WG kein ausgeprägtes wirtschaftliches Interesse (d.h. führend-verkaufendes und ordnend-verwaltendes Interesse) nachgewiesen werden. Auch durch den Besuch des 6WG in der achten Klassenstufe schien sich kein ausgeprägteres wirtschaftliches Interesse zu entwickeln. Die fachbezogenen Wertüberzeugungen der SuS in Mathematik, Physik, Deutsch und Englisch waren an 6WG im Vergleich zu denjenigen an AG unauffällig, sowohl was die Eingangsunterschiede zu Schuljahresbeginn als auch was die Sozialisationseffekte bis zum Ende der achten Klassenstufe anbelangt.

\subsection{Grenzen der Studie}

Bei der Interpretation dieser Befunde sind einige Einschränkungen zu berücksichtigen: Die Selektionseffekte wurden kurz nach Beginn der achten Klassenstufe erfasst. Es ist davon auszugehen, dass bereits die Auseinandersetzung mit den Möglichkeiten der 6BG-Wahl und die Wahl selbst einen Einfluss auf die berichteten Interessenausprägungen und Wertüberzeugungen haben. Mögliche Unterschiede zwischen den Schultypen, die darauf zurückzuführen sind, konnten mit der vorliegenden Studie nicht analysiert werden. Um dies zu erfassen wäre eine Studie nötig, die bereits in der siebten Klassenstufe eine repräsentative Stichprobe von SuS an AG und Realschulen berücksichtigt - bzw. auch Haupt-/Werkreal-SuS mit erhebt. Wechsel von Haupt-/Werkrealschulen an 6BG scheinen jedoch selten zu sein. Um dazu aussagekräftige Ergebnisse erhalten zu können wären qualitative Befragungen oder ein Oversampling nötig.

Im Rahmen der Studie konnte es auch nicht geleistet werden, die Interessen und Wertüberzeugungen von SuS an 6ESG zu untersuchen. Schließlich darf der Hinweis nicht fehlen, dass nur die Entwicklung in der achten Klassenstufe betrachtet werden konnte. Wie sich die Interessen und Wertüberzeugungen bis zum Abitur entwickeln und welchen Einfluss sie auf die schulische Leistung und Übergangsentscheidungen in Beruf und Studium haben, ist ein weiteres noch offenes Forschungsdesiderat, das sich zu untersuchen lohnt. 


\subsection{Fazit}

Mit den 6BG soll SuS schon in der Mittelstufe ein möglichst passgenauer und den eigenen Neigungen und Interessen entsprechender Weg zur Hochschulreife ermöglicht werden und sie sollen dadurch besser als „Fachkräfte von morgen“ gefördert werden (Kultusministeriums Baden-Württemberg 2011). Auch bei Berücksichtigung ihrer Grenzen sprechen die Befunde der vorliegenden Studie dafür, dass die Passung von fachlichem Schwerpunkt zu den persönlichen Neigungen und Interessen der SuS nicht bei allen 6BG gleichermaßen gegeben ist. Bei technischem Schwerpunkt scheint die Passung einfacher zu gewährleisten $\mathrm{zu}$ sein als bei wirtschaftlichem Schwerpunkt.

Hinweise darauf, weshalb es zu diesen unterschiedlichen Resultaten für beide Schulformen kommt, geben die Schulwahlmotive. Die SuS, die die siebte Klassenstufe auf einem AG belegten, haben folgende Angaben zu ihren Wechselgründen auf ein 6BG gemacht: Von denjenigen, die an ein 6TG wechselten und die Frage zum Wechselgrund beantworteten $(n=57)$, gaben $43,9 \%$ an, aus Interesse gewechselt zu haben, 22,8 \% um das Abitur in 13 Schuljahren machen zu können (G9-Modell), und 10,5\% um bessere Noten zu erzielen. Von denjenigen, die vom AG an ein $6 \mathrm{WG}$ wechselten und die Frage zum Wechselgrund beantworteten $(n=72)$, wurde der Wunsch, das Abitur in 13 Schuljahren machen zu können, am häufigsten angegeben $(45,8 \%)$, gefolgt vom Wunsch bessere Noten zu erzielen (20,8\%). Interesse als Wechselgrund wurde nur von 5,6\% genannt (vgl. dazu auch Kramer 2015).

Die 6TG-Wahl scheint also maßgeblich interessengeleitet zu erfolgen, nicht aber die 6WG-Wahl. Deshalb stellt sich die Frage, wie es gelingen kann, Jugendliche stärker für wirtschaftliche Themen zu begeistern. Ob das Fach Wirtschaft, das in Baden-Württemberg ab dem Schuljahr 2016/17 verpflichtend in allen weiterführenden Schulen eingeführt wird, dazu einen Beitrag leistet, kann in den folgenden Jahren beobachtet werden.

Aber auch die anderen Wahlmotive sind opportun. Sie verdeutlichen, dass 6BG bereits in der Sekundarstufe I zur Durchlässigkeit im Bildungssystem beitragen. Es lohnt sich deshalb, 6BG in der Bildungsforschung Beachtung zu schenken - auch um die Frage zu klären, wie groß ihr Beitrag dazu ist, „Fachkräfte von morgen“ gut zu fördern. Gut möglich, dass das ein Kriterium ist, bei dem die Stärken von Wirtschaftsgymnasien deutlich sichtbar werden.

Danksagung Unser Dank gilt dem Mitinitiator der Studie, Stefan Ranzinger, dem Rotary-Club Bietigheim-Vaihingen für die finanzielle Unterstützung, Norman Rose und Wolfgang Wagner für ihre Hilfe bei den Auswertungen sowie Anna Benning, Anna-Lena Dicke, Eve Sarah Müller und Nadja Schröder, die bei der Durchführung der Studie mitwirkten.

Open Access Dieser Artikel wird unter der Creative Commons Namensnennung 4.0 International Lizenz (http://creativecommons.org/licenses/by/4.0/deed.de) veröffentlicht, welche die Nutzung, Vervielfältigung, Bearbeitung, Verbreitung und Wiedergabe in jeglichem Medium und Format erlaubt, sofern Sie den/die ursprünglichen Autor(en) und die Quelle ordnungsgemäß nennen, einen Link zur Creative Commons Lizenz beifügen und angeben, ob Änderungen vorgenommen wurden. 


\section{Literatur}

Barnett, R.C. (1975). Sex differences and age trends in occupational preferences and occupational prestige. Journal of Counseling Psychology, 22(1), 35-38.

Becker, G. S. (1982). Der ökonomische Ansatz zur Erklärung menschlichen Verhaltens. Tübingen: Mohr.

Bergmann, C. (2005). Allgemeiner Interessen-Struktur-Test mit Umwelt Struktur-Test (AIST-R/UST-R). Manual (3. Aufl.). Göttingen: Beltz.

Bergmann, C., \& Eder, F. (1992). AIST/UST Allgemeiner-Interessen-Struktur-Test/Umwelt-Struktur-Test. Weinheim: Beltz.

Bourdieu, P. (1970). Der Habitus als Vermittlung zwischen Struktur und Praxis. In P. Bourdieu (Hrsg.), Zur Soziologie der symbolischen Formen (S. 125-158). Frankfurt am Main: Suhrkamp.

Daniels, Z. (2008). Entwicklung schulischer Interessen im Jugendalter. Münster: Waxmann.

Dreer, B. (2013). Kompetenzen von Lehrpersonen im Bereich Berufsorientierung: Beschreibung, Messung und Förderung. Wiesbaden: SpringerVS.

Eccles, J.S. (2005). Subjective task values and the Eccles et al. model of achievement related choices. In A. J. Elliott \& C.S. Dweck (Hrsg.), Handbook of competence and motivation (S. 105-121). New York: Guilford Press.

Eder, F. (1988). Die Auswirkungen von Person-Umwelt-Kongruenz bei Schülern: Eine Überprüfung des Modells von J. L. Holland. Zeitschrift für Pädagogische Psychologie, 2, 259-270.

Eder, F., \& Reiter, C. (2002). Interessen und Schullaufbahn. In C. Wallner-Paschon \& G. Haider (Hrsg.), PISA Plus 2000. Thematische Analysen nationaler Projekte (S. 11-116). Innsbruck: StudienVerlag.

Gaspard, H., Dicke, A.-L., Flunger, B., Schreier, B., Häfner, I., Trautwein, U., \& Nagengast, B. (2015). More value through greater differentiation: gender differences in value beliefs about math. Journal of Educational Psychology, 107(3), 663-677.

Gottfredson, L.S. (1981). Circumscription and compromise: A developmental theory of occupational aspiration. Journal of Counseling Psychology, 28(6), 545-579.

Gottfried, A. E., Fleming, J. S., \& Gottfried, A. W. (2001). Continuity of academic intrinsic motivation from childhood through late adolescence: A longitudinal study. Journal of Educational Psychology, 93(1), $3-13$.

Häußler, P., \& Hoffmann, L. (1995). Physikunterricht - an den Interessen von Mädchen und Jungen orientiert. Unterrichtswissenschaft, 23(2), 107-126.

Heller, K. A., \& Perleth, C. (2000). KFT 4-12+R Kognitiver Fähigkeitstest für 4. bis 12. Klassen, Revision - Manual. Weinheim: Beltz.

Holland, J. L. (1997). Making vocational choices, a theory of vocational personalities and work environments (3. Aufl.). Odessa FL: Psychological Assessment Resources.

Kohlberg, L. (1967). A cognitive-developmental analysis of children's sex-role concepts and attitudes. In E. E. Maccoby (Hrsg.), The development of sex differences (S. 82-173). London: Travistock.

Köller, O., Watermann, R., \& Trautwein, U. (2004). Transformation des Sekundarschulsystems in der Bundesrepublik Deutschland: Differenzierung, Öffnung von Bildungswegen und die Wahrung von Standards. In O. Köller, R. Watermann, U. Trautwein, \& O. Lüdtke (Hrsg.), Wege zur Hochschulreife in Baden-Württemberg (S. 13-27). Opladen: Leske + Buderich.

Köller, O., Trautwein, U., Lüdtke, O., \& Baumert, J. (2006). Zum Zusammenspiel von schulischer Leistung, Selbstkonzept und Interesse in der gymnasialen Oberstufe. Zeitschrift für Pädagogische Psychologie, 20(1/2), 27-39.

Kramer, J. (2015). Berufliche Gymnasien: Entwicklung, Effekte und aktuelle Herausforderungen. Recht der Jugend und des Bildungswesens, 63(3), 257-270.

Krapp, A. (1998). Entwicklung und Förderung von Interessen im Unterricht. Psychologie in Erziehung und Unterricht, 44(3), 185-201.

Kultusministerium Baden-Württemberg (2011). 15 пеие sechsjährige Berufliche Gymnasien starten zum Schuljahr 2012/13. Stuttgart: Kultusministerium Baden-Württemberg.

Kultusministerium Baden-Württemberg (2012a). Lehrplan für das berufliche Gymnasium der sechsjährigen Aufbauform, Technische Richtung, Technik, Klasse 8, 9 und 10. http://www.ls-bw.de/ bildungsplaene/beruflschulen/bg/bg_berufsbezogen/Mittelstufe/TG. Zugegriffen: 4. Mai 2017.

Kultusministerium Baden-Württemberg (2012b). Lehrplan für das berufliche Gymnasium der sechsjährigen Aufbauform, Wirtschaftswissenschaftliche Richtung, Volks- und Betriebswirtschaftslehre, Klasse 8, 9 und 10. http://www.ls-bw.de/bildungsplaene/beruflschulen/bg/bg_berufsbezogen/Mittelstufe/ WG. Zugegriffen: 4. Mai 2017. 
Kultusministerium Baden-Württemberg (2015). Berufliche Gymnasien. http://www.kultusportal-bw.de/, Lde/Startseite/schulebw/Berufliche+Gymnasien. Zugegriffen: 4. Mai 2017.

Lüdtke, O., \& Trautwein, U. (2004). Die gymnasiale Oberstufe und psychische Ressourcen: Gewissenhaftigkeit, intellektuelle Offenheit und die Entwicklung von Berufsinteressen. In O. Köller, R. Watermann, U. Trautwein, \& O. Lüdtke (Hrsg.), Wege zur Hochschulreife in Baden-Württemberg (S. 367-401). Opladen: Leske + Buderich.

Lüdtke, O., Robitzsch, A., Trautwein, U., \& Köller, O. (2007). Umgang mit fehlenden Werten in der psychologischen Forschung. Psychologische Rundschau, 58(2), 103-117.

Maaz, K., Baumert, J., \& Trautwein, U. (2011). Genese sozialer Ungleichheit im institutionellen Kontext der Schule: Wo entsteht und vergrößert sich soziale Ungleichheit? In H.-H. Krüger, U. Rabe-Kleberg, R.-T. Kramer, \& J. Budde (Hrsg.), Bildungsungleichheit revisited. Bildung und soziale Ungleichheit vom Kindergarten bis zur Hochschule (2. Aufl., S. 69-102). Wiesbaden: SpringerVS.

Muthén, L. K., \& Muthén, B. O. (2012). Mplus - Statistical analysis with latent variables (7. Aufl.). Los Angeles CA: Muthén \& Muthén.

Rheinberg, F. (1981). Leistungsmotivation. In H. Schiefele \& A. Krapp (Hrsg.), Handlexikon zur Pädagogischen Psychologie (S. 227-231). München: Ehrenwirth.

Suter, P. (2013). Determinanten der Schulwahl: Elterliche Motive für oder gegen Privatschulen. Wiesbaden: SpringerVS.

Todt, E., \& Schreiber, S. (1998). Development of interests. In L. Hoffmann, A. Krapp, K. A. Renninger \& J. Baumert (Hrsg.), Interest and learning. Proceedings of the Seeon Conference on Interest and Gender (S. 25-40). Kiel: Institute for Science Education at the University of Kiel (IPN).

Travers, R. M. W. (1978). Children's interest. Kalamazoo: Michigan University.

Watermann, R., \& Maaz, K. (2006). Effekte der Öffnung von Wegen zur Hochschulreife auf die Studienintention am Ende der gymnasialen Oberstufe. Zeitschrift für Erziehungswissenschaft, 9(2), 219-239. 